\title{
Chronic maxillary sinusitis: Clinical and microbiological evaluation
}

D. Shrestha ${ }^{1}$, L. K. Yadav ${ }^{2}$, P. Thapa ${ }^{3}$

${ }^{1}$ Assistant Professor, ${ }^{2}$ Sr. Consultant, ${ }^{3}$ Registrar, Department of ENT and Head \& Neck Surgery, Bir Hospital, NAMS, Kathmandu, Nepal.

\begin{abstract}
Chronic sinusitis essentially results from untreated or inadequately treated acute sinusitis. Sinusitis is one of the common health problems worldwide. This is a prospective study, done in Department of ENT Bir Hospital Kathmandu. The study period was one year from 14 March 2009 to 15 March 2010. In this study the most commonly involved group is $21-30$ years (44\%). The most presenting symptoms was Nasal discharge $46(92 \%)$ and nasal obstruction $44(88 \%)$. The most common sign was mucopus in nasal cavity in $44(88 \%)$ cases followed by post nasal drip in $39(78 \%)$ cases. The bacteria most frequently isolated from sinus aspirates were staphylococcus aureus 18(36\%) and streptococcus pneumonia 16(32\%). Majority of the aspirates $34(68 \%)$ cases yielded single organism. The antibiotic sensitivity testing showed that $48(96 \%)$ cases of isolates were sensitive to Cephalexin and ceftriaxone.
\end{abstract}

Key words: Chronic sinusitis, bacteriology, culture sensitivity.

\section{Introduction}

Sinusitis is defined as the inflammation of the mucosal lining of the paranasal sinuses. ${ }^{1}$ Irrespective of whether acute or chronic, the sequences of events in pathogenesis of sinusitis are based on the ability of the bacteria to replicate and the host defence to overcome the bacterial growth. The course passes through two phases, initial viral or allergic followed by bacterial stage. ${ }^{2}$ Chronic sinusitis essentially results from untreated or inadequately treated acute sinusitis. The most commonly affected sinus is the maxillary antrum sinus. Nasal obstruction, headache with midfacial pain, cough, nasal discharge and postnasal drip are the most common presenting features. If the

Correspondence: D. Shrestha

E-mail: shrestha_deependra@yahoo.com duration persists more than three months it is called chronic sinusitis. Lanza DC and Kennedy D.W. had categorized the symptom complex under major and minor criteria. Major criteria included purulent nasal discharge, headache, facial pain, nasal blockage and decreased smell sensation. Minor criteria included halitosis, fever, weakness, dental pain, fullness in the ear and cough. According to the Authors, if two or more of the above mentioned major criteria or one major and two minor criteria are present for more than 12 weeks chronic maxillary sinusitis is likely. ${ }^{3}$

Sinusitis is one of the common health problems worldwide. In USA, sinusitis affects 31 million people per year. ${ }^{4}$ It has been estimated that $5 \%$ of 
the urban population and $15 \%$ of the total population suffer from chronic sinusitis. ${ }^{5}$ Sinusitis is also one of the common presentations in the ENT OPD of Bir Hospital, Kathmandu. Chronic maxillary sinusitis is a disease with rising prevalence that costs society millions of rupees and accurate. Therefore easy and safe method of diagnosing the disease and planning its management are essential. The present study is focused on identifying the cases of chronic maxillary sinusitis by setting a fixed clinical criteria followed by an attempt to find out various bacteria associated with the conditions and the most sensitive drugs to the bacteria isolated.

\section{Materials and methods}

This is a prospective study. This study was done in Department of ENT and Head and Neck Surgery, Bir Hospital Kathmandu. The study period was one year from 14 March 2009 to 15 March 2010. This study was conducted to see the common bacteria associated with chronic maxillary sinusitis in our population and to correlate the clinical findings and predisposing conditions such as age, sex, allergy and other abnormalities. It was also intended to determine the sensitivity pattern of organisms associated with chronic maxillary sinusitis to chemotherapeutic agents in common use. Fifty patients were included in this study. Those patients to whom antral puncture could not be performed were not included in this study. Patients with minimum three symptoms for more than three months and one sign with positive $\mathrm{x}$-ray paranasal sinus findings (haziness opacity, fluid level in maxillary sinus) were clinically diagnosed as chronic maxillary sinusitis and included in this study. Detail history of the all included patients was taken, detail ENT examinations were done and findings were noted in a proforma prepared for this study. Bilateral antral wash was done in Out Patient Department with all asceptic precautions under local anaesthesia. The aspirated samples were sent to the microbiology department for culture and sensitivity test.

\section{Results}

Maximum number of cases were found between the ages 21-30 years 22(44\%) and 31-40 years15 (30\%) followed by $11-20$ years $10(20 \%), 41-55$ years $3(6 \%)$.

In the presenting symptoms Nasal discharge 46(92\%), nasal obstruction 44(88\%), Headache or Facial pain34 (68\%) followed by post nasal drip33 (66\%), hyposmia9 (18\%), and epistaxis $3(6 \%)$.

The most common associated sign was mucopus in the nasal cavity (middle meatus) $44(88 \%)$ cases, followed by post nasal drip 39(78\%), tenderness in the maxillary sinus34 (68\%), deviated nasal septum 14(28\%), and dental infection 3(6\%) cases. The bacteria most frequently isolated from sinus aspirates were staphylococcus aureus 18(36\%), streptococcus pneumonia 16(32\%), followed by Haemophilus influenza 7(14\%) and Pseudomonas aeruginosa $4(8 \%)$. The other bacteria isolated were staphylococcus epidermidis 3(6\%), and Streptococcus viridians $3(6 \%)$. There was no growth in $9(18 \%)$ cases of aspirates.

Majority of the aspirates 34(68\%) cases yielded single organism, while in 5(10\%) cases of the 
D. Shrestha et al. Chronic maxillary sinusitis: Clinical and microbiological evaluation

aspirates two organisms and in $2(4 \%)$ cases three types of organism were isolated. No growth was isolated in $9(18 \%)$ cases.

The antibiotic sensitivity testing showed that $48(96 \%)$ cases of isolates were sensitive to Cephalexin and ceftriaxone and only $2(4 \%)$ cases were resistant. Similarly, Doxycycline was found to be sensitive in $43(86 \%$ ) cases of isolates followed by Amoxycillin in 38(76\%) cases, Erythromycin in $37(74 \%$ ) cases, Ciprofloxacin in 34(68\%) cases and, Cotrrimoxazole 28(56\%) cases.

Table No.1: Age and Sex distribution

\begin{tabular}{lccc}
\hline Age & Male & Female & Total \\
\hline 0-10 years & 0 & 0 & 0 \\
11-20 years & 7 & 3 & 10 \\
21-30 years & 13 & 9 & $22(44 \%)$ \\
31-40 years & 7 & 8 & $15(30 \%)$ \\
41-55 years & 1 & 2 & $3(6 \%)$ \\
Total & $\mathbf{2 8}$ & $\mathbf{2 2}$ & $\mathbf{5 0}(\mathbf{1 0 0 \%})$ \\
\hline
\end{tabular}

Table No. 2: Presenting symptoms

\begin{tabular}{lc}
\hline Symptoms & Numbers \\
\hline Nasal discharge & $46(92 \%)$ \\
Nasal obstruction & $44(88 \%)$ \\
Headache/Facial pain & $34(68 \%)$ \\
Post nasal drip & $33(66 \%)$ \\
Cough & $21(42 \%)$ \\
Hyposmia & $9(18 \%)$ \\
Epistaxis & $3(6 \%)$ \\
\hline
\end{tabular}

Table No. 3: Various signs of chronic maxillary sinusitis

\begin{tabular}{|c|c|}
\hline Signs & Number of cases \\
\hline \multicolumn{2}{|l|}{ Mucopus in nasal cavity } \\
\hline (middle meatus) & $44(88 \%)$ \\
\hline Post nasal drip & $39(78 \%)$ \\
\hline Tenderness in max. sinus & $34(68 \%)$ \\
\hline Deviated Nasal Septum & $14(28 \%)$ \\
\hline Dental infection & $03(6 \%)$ \\
\hline
\end{tabular}

Table No.4: Bacteria isolated from maxillary sinusitis aspirates

\begin{tabular}{lcc}
\hline Bacteria isolated & No. of cases & Percentage \\
\hline Staphylococcus aureus & 18 & $36 \%$ \\
Streptococcus pneumoniae & 16 & $32 \%$ \\
Haemophilus influenzae & 7 & $14 \%$ \\
Pseudomonas aeruginosa & 04 & $8 \%$ \\
Staphylococcus epidermidis & 3 & $6 \%$ \\
Streptococcus viridans & 3 & $6 \%$ \\
No growth & 9 & $18 \%$ \\
\hline
\end{tabular}

Table No. 5 : Antibiotic Sensitivity

\begin{tabular}{lcc}
\hline Antibiotic & Sensitive & Resistant \\
\hline Cephalexin & $48(96 \%)$ & $2(4 \%)$ \\
Ceftriaxone & $48(96 \%)$ & $2(4 \%)$ \\
Doxycycline & $43(86 \%)$ & $7(14 \%)$ \\
Amoxycillin & $38(76 \%)$ & $12(24 \%)$ \\
Erythromycin & $37(74 \%)$ & $13(26 \%)$ \\
Ciprofloxacillin & $34(68 \%)$ & $16(32 \%)$ \\
Cotrimoxazole & $28(56 \%)$ & $22(44 \%)$ \\
\hline
\end{tabular}




\section{Discussion}

Sinusitis is a disease faced by otorhinolaryngologists frequently. The large number of patients with chronic sinusitis is treated without proper investigations and specialist's opinion. The otorhinolaryngologists is likely to see only those cases of sinusitis that have failed to respond to treatment, those with incipient or actual complications. A persistent mucopurelent discharge with associated coughing, pharyngeal irritation or facial pain may exist in combination or individually. Symptoms may vary although nasal obstruction, hyposmia and occasionally cachosmia may occur. ${ }^{6}$ Under these circumstances further investigations may be appropriate and study of microbial flora by antral puncture can help immensely in guiding the direction of management. Once the probable organisms are isolated and their antibiotic sensitivity pattern determined, treatment can be started according to the sensitivity result, which will be more scientific than blindly starting antibiotic without doing a culture sensitivity test. Thus the relevance of the study of microbial flora of chronic maxillary sinusitis is obvious if the treatment is instituted on a scientific pathological basis.

In this study the most commonly involved group is 21-30years $22(44 \%)$ cases followed by 31-40 years $15(30 \%)$ cases. However in a similar study done by Akhund $^{6}$ maximum number of cases were found between the ages of 11-20 yrs (34\%) and 21-30 years (47\%) followed by 31-40 yrs $8 \%$, 41$50 \mathrm{yrs} 8 \%, 50 \mathrm{yrs}$ and above $2 \%$ and only one cases was less than 10 years of age.
The most common symptoms was nasal discharge $46(96 \%)$ cases followed by nasal obstruction 44(88\%), headache/ facial pain 34(68\%) cases, post nasal drip 33(66\%) cases cough 21(42\%) cases, hyposmia $9(18 \%)$ cases, epistaxis $3(6 \%)$ cases. The most common sign was mucopus in nasal cavity in $44(88 \%)$ cases followed by post nasal drip in $39(78 \%)$ cases, tenderness in maxillary sinus in $34(68 \%)$ cases, deviated nasal septum in14(28\%) cases and dental infection in 3(6\%) cases. Damnl ${ }^{7}$ found nasal obstruction $92 \%$, post nasal drip $87 \%$ as the leading symptoms in their study. Akhund ${ }^{6}$ found the common presenting symptoms were nasal discharge (96\%), nasal obstruction 93\%, followed by facial pain $30 \%$, Headache $19 \%$, and sneezing $19 \%$.Von Dishoeck and Franssen ${ }^{8}$ demonstrated nasal allergy in $60 \%$ cases. Catlin et $\mathrm{al}^{9}$ observed nasal discharge in $98 \%$ of cases, facial pain in $66 \%$, history of nasal allergy in $20 \%$, deviated nasal septum in $20 \%$ cases.

The commonest organisms isolated in this study were staphylococcus aureus 18(36\%) and streptococcus pneumonia 16(32\%) followed by Haemophilus influenza 7(14\%), Pseudomonas aeruginosa4 (8\%), staphylococcus epidermidis $3(6 \%)$ and Streptococcus viridians3 $(6 \%)$ and no growth obtained in $9(18 \%)$ cases. Single organisms were isolated in $34(68 \%)$ cases, two organisms were isolated in $5(10 \%)$ cases, three organisms were isolated in $2(4 \%)$ cases and no growth obtained in 9(18\%) cases. Various similar studies have shown varying results regarding the percentage of different bacteria isolated. However in most of the studies staphylococcus has been 
D. Shrestha et al. Chronic maxillary sinusitis: Clinical and microbiological evaluation

found to be the most common organism and in some streptococcus has topped the list. Anwar Ali Akhund $^{6}$ reported Streptococcus pneumonia as the commonest organism (35.4\%) followed by Staph. Aureus (23.6\%), H. influenza (14.5\%). Kinnman etal isolated H. influenza (49\%) and Strep.pneumoniae (29\%) as the common pathogens in cases of chronic maxillary sinusitis. Nicolas Y. Busaba $^{10}$ isolated coagulase negative Staphylococcus (53\%) most frequently followed by Staphylococcus aureus (18\%).

The antibiotic sensitivity pattern showed cephalexin and ceftriaxone to be the most effective. $48(96 \%)$ of the isolates were sensitive to cephalexin and ceftrixone, 43(86\%) to Doxycycline, 38(76\%) to Amoxycillin, 37(74\%) to Erythromycin, 34(68\%) to Ciprofloxacin and 28(56\%) to Cotrimoxazole. These 11 findings are correlated with the observations of Anwar Ali Akhund ${ }^{6}$ who observed 90-98\% sensitivity with Cephalosporin group of antibiotic and Doxycycline and 30-35\% resistance with Cotrimoxazole and Ciprofloxacin. These findings correlate with the observations of Gwaltney et $\mathrm{al}^{11}$ and Shahdin et $\mathrm{al}^{12}$ who observed $30-50 \%$ resistance with Erythromycin and Cotrimoxazole.

\section{Conclusion}

Chronic Sinusitis is more common in the 21-30 years of age group. Nasal discharge and Nasal obstruction are the most common symptoms associated with the chronic maxillary sinusitis. The most common sign was mucopus in nasal cavity followed by post nasal drip. The most frequently isolated bacteria in this study were Staphylococcus,
Streptococcus pneumonia and H. influenza. Antibiotic treatment should cover these organisms if started empirically before culture sensitivity report of the aspirate is available. The most sensitive drugs to the bacterial isolates in this study were Cephalexin, Ceftrixone, and Doxycycline.

\section{References}

1. N.Weir, D.G. Golding Wood; Infective rhinitis and sinusitis. Scott Brown`s otolaryngology.1974;8:1-45.

2. C. Carenfelt. The pathogenesis of Sinus empyema. Ann. Otol.Rhinol.laryngol., 1979; 88:16-20.

3. D.C. Lanza, D.W. Kennedy; Adult rhinosinusitis defined. ORL Head and Neck Surgery; 1997; 117: 51-7.

4. W. George. Facer, Eugene B Kern; Sinusitis: Current concept and management. Head and Neck surgery. Otolaryngology. 1998; 33: 441-50.

5. I. Helen, L. Lindahl, L. Anderson. Chronic maxillary sinusitis. Acta Otolaryngology; 1986; 101; 320-7.

6. A.A. Akhund, A.R. Shaikh . Maxillary Sinusitis. Bacterial organisms and antibiotic Sensitivity. Pakistan Journal of Otolaryngology 1993; 9:80-3.

7. M. Damm, G. Quante. Impact of functional endoscpic sinus surgery on symptoms and quality of life in chronic rhinosinusitis. Laryngoscope, 2002;112:310-5.

8. J.A. Van Dishoeck, M.G.C. Franssen. The incidence and correlation of allergy and chronic maxillary sinusitis. Pract. Otrhinolaryngol.1957; 19:502-6.

9. F.A. Catlin, L.E. Cluff, R.C. Reyonalds. The bacteriology of acute and chronic sinusitis. South Med.J., 1965; 55:1497-502. 
Journal of College of Medical Sciences-Nepal,2011, Vol-7,No-2

10. Y. N. Busaba, N. Siegel. Bacteriology of nontraumatic maxillary sinus mucoceles versus chronic sinusitis. Laryngoscope, 2000;110: 969-71

11. J.M. Gwalteny, A. Syndor, M.A. Sandi. Etiology and antimicrobial treatment of acute sinusitis. Ann.Otol. Rhinol Laryngol.(Supp.)1981;90:68-71.
12. K. Shahin, H. Afzal , M. Asfaque et al. Incidence and antibiotic sensitivity of Staphylococci in infected ear, nose and throat.J.P.M.A.1967;29:58-61. 